\footnotetext{
Mnil

Faculté de biologie

et de médecine

éventuelle
}

Logo d'une autre institution

(CHUV, UNIGE, HES-SO, etc.)

Mémoire de Maîtrise en médecine

\title{
Postmortem IgE determination in coronary artery disease
}

\author{
Etudiant \\ Lambert Karine \\ Tuteur \\ Palmiere Cristian \\ Expert \\ Bart Pierre-Alexandre
}

Lausanne, 14.01.19 
Postmortem IgE determination in coronary artery disease

\begin{abstract}
Allergic inflammation is thought to play a role in atherogenesis and atherosclerotic disease progression. IgE may activate mast cells and macrophages located within atheromatous lesions, which may contribute to local inflammation and disease progression. On the other hand, increased lgE levels in individuals with acute myocardial infarction might also be the consequence of immunological reactions to damaged coronary arteries or myocardium. In this study, total IgE and mast cell tryptase were measured in a series of forensic autopsy cases showing various degrees of coronary artery disease, in order to correlate laboratory results with morphological findings and compare them to conclusions reported in the clinical setting. A total of 84 subjects were selected and two study groups were retrospectively formed, a non-allergic, cardiac death group (42 cases) and a non-allergic, non-cardiac control group (42 cases). Our findings indicated that a portion of acute coronary syndromes and coronary deaths characterized by coronary atherosclerotic lesions of various severities are also characterized by increased total IgE and mast cell tryptase levels, thus corroborating the data previously reported in both clinical and forensic literature on this topic as well as the necessity of combining morphological investigations focusing on the heart and coronary arteries with biochemical analyses.
\end{abstract}

Keywords: Coronary Artery Disease; IgE; Postmortem Biochemistry; Forensic; Autopsy

\title{
Introduction
}

Inflammation has been shown to play a significant role in atherosclerosis and atherosclerotic cardiovascular disease development. Atheromatous lesions are characterized by inflammatory cell infiltrates, including macrophages, lymphocytes, and mast cells as well as others. Cytokines, chemokines, proinflammatory mediators, proteases, and other products of these cells are likely involved in lesion development and progression. In addition, a special type of inflammation (allergic inflammation) is thought to play a role in atherogenesis and atherosclerotic disease progression $[1,2]$.

Mast cell accumulation has been noted in the shoulder regions of coronary atheromatous lesions and at the actual sites of coronary plaque erosion or rupture. Based on these findings, mast cell activation has been proposed as one of the mechanisms linking allergic inflammation with atherosclerotic disease progressions [1,3-8].

Indeed, numerous biological and clinical studies have postulated the existence of a potential relationship between IgE-induced mast cell degranulation and coronary artery disease. Elevated total IgE levels have been found in individuals with myocardial infarction, suggesting a direct role of IgE, atopy, allergy, and mast cell degranulation in the process $[2,3,9]$.

IgE may activate mast cells and macrophages located within atheromatous lesions by binding to high and low affinity FcعRI and FcERII receptors situated on the surface of these cells. This would therefore activate inflammatory mediator release, which would in turn lead to local inflammation, immunological reaction, local IgE-mediated response, and atheromatous lesion progression. However, higher IgE in individuals with acute myocardial infarction might also be the consequence of immunological reactions due to damaged coronary arteries or myocardium. Thus, findings potentially linking IgE, atopy, and allergic inflammation to atherosclerotic cardiovascular disease in humans cannot be clearly established and have been conflicting [1-3,5,8-10].

In the forensic setting, Edston and van Hage-Hamsten [5] measured mast cell tryptase and total serum IgE in 29 cases of sudden death from coronary artery thrombosis and 27 control cases. Although no statistical difference in mast cell tryptase and total IgE in serum between the groups, they documented $20 \%$ of coronary deaths had elevated IgE values (higher than $200 \mathrm{kU} / \mathrm{l}$ ) compared to only $8 \%$ in the control group. They also observed higher mast cells within coronary artery walls in cardiac deaths. Apart from this study, the forensic literature has limited reports on mast cell tryptase and total serum lgE in atherosclerotic cardiovascular disease. 
The presented study compared postmortem total IgE and mast cell tryptase collected from femoral blood between autopsy cases with various degrees of coronary artery atherosclerosis and cases control without coronary artery atherosclerosis.

Material and methods

Study design and study populations

A 10-year, case controlled, retrospective study between 2008 and 2018 (inclusive) was performed. All selected cases originated from forensic practice and underwent forensic investigations as requested by local inquiring authorities (the public prosecutor). Postmortem biochemical analyses (IgE and mast cell tryptase determination) were performed as part of routine forensic investigations. All forensic autopsies were jointly performed by two forensic pathologists (at least one board-certified), as in accordance with both local standards and international guidelines for medicolegal cases.

Case and control selection

Cases where the postmortem interval (defined as the interval between death and femoral blood sampling) did not exceed 48 hours, no history of atopy and full complete autopsy performed including histology, toxicology, and postmortem biochemical analyses on femoral blood (IgE and mast cell tryptase determination) were initially identified.

The study was designed such that the same number of cases were used for both case and control group and within each group having an equal spread of disease severity. A total of 84 cases ( 71 males and 13 females), with a mean age of 49.6 years (range 19-58 years), were identified. The cases were initially divided into two groups of equal numbers. The study group (42 cases) was non-allergic cardiac, the control group (42 cases) was nonallergic non-cardiac deaths.

Non-allergic cardiac death group $(n=42)$

14 non-allergic, cardiac deaths were apparently healthy individuals who died suddenly in the presence of witnesses, with minimal or no coronary atherosclerosis. Autopsies and histology failed to reveal plaque ruptures, plaque hemorrhages or luminal thrombi. Some degree of coronary atherosclerosis consisting of lipid plaques was sporadically observed. Based on the results of all forensic investigations, the cause of death in all these cases was considered cardiac arrhythmia and cardiac arrest in the absence of macroscopic and microscopic abnormalities other than minimal coronary artery atherosclerosis.

Another 14 cases had significant coronary artery atherosclerosis, coronary artery calcifications, and severe luminal narrowing as well as myocardial fibrosis of various degrees, without evidence of acute coronary thrombosis or myocardial infarction. Autopsies and histology revealed coronary atherosclerotic plaques leading to partial or complete luminal stenosis as well as various extents of myocardial fibrosis. The presence of ruptured plaques and luminal thrombi was histologically excluded in all cases. In addition, mast cell accumulation and other inflammatory cells were noted within atherosclerotic plaques. Based on the results of all forensic investigations, the cause of death in all these cases was determined to be cardiac arrest due to severe coronary artery atherosclerosis and myocardial fibrosis.

The last 14 cases of this group concerned individuals with significant coronary artery atherosclerosis, acute coronary thrombosis, and acute myocardial infarction. Autopsies and histology revealed ruptured plaques with acute, superimposed luminal thrombi. Accumulation of mast cells and other inflammatory cells was noticed within atherosclerotic plaques. The cause of death was attributed to cardiac arrest due to myocardial infarction in all these cases.

Non-allergic non-cardiac death $(n=42)$ 
The 42 non-allergic, non-cardiac deaths selected for the control group consisted of 21 cases of hanging as cause of death and 21 of intracranial gunshot wounds. All these were males and had minimal or no coronary artery atherosclerosis at autopsy.

Postmortem investigations and sample collection

At autopsy the hearts were sectioned either before or after fixation in $10 \%$ neutral buffered formalin. The major epicardial coronary arteries were either serially sectioned at approximately 2-3 mm intervals or longitudinally sectioned intact on the heart. In selected cases, segments of interest were removed from the heart and retained for decalcification if needed before paraffin processing.

Conventional histology included haematoxylin-eosin (HE) stain of the brain, heart, coronary arteries, lung, liver, and kidneys. HE stain was performed after tissue fixation in formaldehyde.

Sections of the left and right heart ventricles were examined for evidence of ischemia or infarction. Full thickness areas involving the left anterior ventricle, the lateral free wall of the left ventricle, left posterior ventricle, interventricular septum and right anterior ventricle were systematically sampled.

In selected cases, coronary arteries (with or without thrombi) were serially embedded in paraffin and divided into segments maintaining proximal-to-distal orientation. Histological sections were prepared at three different, equally spaced levels to best identify plaque rupture sites. Routine histology stains for heart tissues and coronary arteries included HE and Masson's trichrome.

Peripheral blood from femoral veins was systematically collected as soon as possible upon arrival of the bodies at the morgue and prior to autopsy. Blood samples were drawn by aspiration with sterile needles after clamping the vein(s) at the proximal end and lifting the lower limb(s) for several minutes. Samples were stored in preservative free gel serum separator tubes that were centrifuged immediately post collection at $3000 \mathrm{~g}$ for $15 \mathrm{~min}$. After centrifugation, the separated supernatant (postmortem serum) was collected and stored in preservative free tubes. Postmortem serum samples were transferred to the laboratories immediately post collection. When analyses were delayed, samples were stored at $-20^{\circ} \mathrm{C}$.

Laboratory assays

Biochemical investigations systematically included mast cell tryptase and total IgE measurement in postmortem serum from femoral blood using a commercial fluoroenzyme immunoassay method (Pharmacia \& Upjohn, Fisher Scientific, Thermo Fisher Scientific, Pittsburgh, PA, USA).

For the purpose of this study, increased total lgE in postmortem serum from femoral blood was defined as exceeding the highest upper limit of the clinical reference value indicated by the clinical laboratory where the analysis was performed (5-50 kU/l).

Analogously, increased mast cell tryptase in postmortem serum from femoral blood was defined as exceeding the highest upper limit of the clinical reference value indicated by the clinical laboratory where the analysis was performed $(13 \mathrm{ng} / \mathrm{ml})$. Detection limit was below $2 \mathrm{ng} / \mathrm{ml}$.

\section{Statistical analyses}

Data were analyzed by using Greaph Pad Prism (Graph Pad Software Inc.). Postmortem serum total IgE and tryptase levels among groups were compared using non-parametric tests. Statistical significance was set at $p<0.05$.

Ethical issue

All relevant ethical issues were identified and discussed with the local Ethical Committee. All cases included in this study underwent forensic autopsies as requested by the inquiring authorities. Postmortem serum samples are routinely collected during autopsy for 
toxicological and/or biochemical purposes in our facility. Moreover, postmortem biochemical analyses are routinely performed as part of forensic investigations. All biological samples were anonymized prior to analysis and analyzed in the same laboratory. No ethical approval was necessary to perform biochemical analyses in the collected cases.

\section{Results}

In non-allergic cardiac death cases with minimal or no coronary artery atherosclerosis, postmortem serum total IgE levels were lower than the clinical reference value in 12 out of 14 cases.

Total IgE ranged between 11 and $48 \mathrm{kU} / \mathrm{l}$ (in the 12 negative cases). Two cases had total IgE of $289 \mathrm{kU} / \mathrm{l}$ and $316 \mathrm{kU} / \mathrm{l}$ (the 2 positive cases were a 46-year-old male and a 51-year-old female). Postmortem serum mast cell tryptase levels were lower than clinical reference values in all cases included in this group.

In non-allergic cardiac death cases with significant coronary artery atherosclerosis but without evidence of acute coronary thrombosis/acute myocardial infarction, postmortem serum total IgE levels were lower than the clinical reference value in 12 out of 14 cases.

Total IgE values ranged between 13 and $44 \mathrm{kU} / \mathrm{l}$ (in the 12 negative cases). Two cases had IgE of $351 \mathrm{kU} / \mathrm{l}$ and $442 \mathrm{kU} / \mathrm{l}$ (the 2 positive cases were both males, 49 and 55 years of age respectively). Postmortem serum tryptase levels were lower than the clinical reference value in 13 out of the 14 cases. The only mast cell tryptase positive case (value $35 \mathrm{ng} / \mathrm{ml}$ ) was observed in the case that had total IgE values at $442 \mathrm{kU} / \mathrm{l}$ (55-year-old male). Microscopy performed on spleen samples in this case did not show any evidence of mast cell infiltration or degranulation.

In the group of individuals with significant coronary artery atherosclerosis, acute coronary thrombosis, and acute myocardial infarction, postmortem serum total IgE levels were lower than the clinical reference value in 11 out of 14 cases. Total IgE values ranged between 9 and $49 \mathrm{kU} / \mathrm{l}$ (in the 11 negative cases). Three cases had lgE of $111 \mathrm{kU} / \mathrm{l}, 241 \mathrm{kU} /$ $\mathrm{l}$, and $264 \mathrm{kU} / \mathrm{l}$ (these 3 positive cases were a 39-year-old male, a 43-year-old male, and a 47-year-old female). Postmortem serum tryptase levels were lower than the clinical reference value in 12 out of the 14 cases. The two mast cell tryptase positive cases (values $39 \mathrm{ng} / \mathrm{ml}$ and $41 \mathrm{ng} / \mathrm{ml}$ ) were observed in those cases that had total lgE values at $241 \mathrm{kU} / \mathrm{l}$ and $264 \mathrm{kU} / \mathrm{l}$ (the 43 -year-old male and 47 year old female). A tryptase level of $12 \mathrm{ng} / \mathrm{ml}$ was noted in the male case with $111 \mathrm{kU} / \mathrm{l}$.

Globally considered, these findings revealed postmortem serum total lgE levels were higher than the clinical reference value in 7 out of 42 cases $(17 \%)$ and mast cell tryptase levels higher than the clinical reference value in 3 out of 42 cases $(7 \%)$.

Non-allergic, non-cardiac death cases had postmortem serum total IgE levels lower than the clinical reference value in 39 out of 42 cases. Total lgE values ranged between 8 and 48 $\mathrm{kU} / \mathrm{l}$. In 3 cases out of 42 (7\%), IgE levels were at 55, 62 and $98 \mathrm{kU} / \mathrm{l}$ (three males of 51, 35 and 29 years of age respectively). Postmortem investigations revealed mild coronary artery atherosclerosis in these three cases (51-year-old male, total IgE $55 \mathrm{kU} / \mathrm{l}$, hanging) and minimal coronary artery atherosclerosis consisting of lipid plaques exclusively (35-year-old male, total IgE $62 \mathrm{kU} / \mathrm{l}$, hanging, and 29 year-old male with total IgE $98 \mathrm{kU} / \mathrm{l}$, gunshot wound). Postmortem serum mast cell tryptase levels were not increased in any of these cases. In addition, microscopy performed on spleen samples failed to show any evidence of mast cell infiltration or degranulation.

Statistically speaking, the highest postmortem serum total IgE values were observed in the group of individuals with significant coronary artery atherosclerosis without evidence of acute coronary thrombosis/acute myocardial infarction $(351 \mathrm{kU} / \mathrm{l}$ and $442 \mathrm{kU} / \mathrm{l})$.

Within the limits of the few IgE and tryptase positive cases observed in the group of cardiac deaths and controls, there was a statistically significant difference in total IgE concentrations between cardiac death cases and controls $(17 \%$ versus $7 \%$ with a mean value of $288 \mathrm{kU} / \mathrm{l}$ versus $71 \mathrm{kU} / \mathrm{l}$ ). As stated above, no statistics could be performed concerning mast cell tryptase levels since tryptase positive cases were observed exclusively in the cardiac death group, with slightly higher tryptase levels in those individuals with acute myocardial infarction. 


\section{Discussion}

Atopy is defined as a familiar or personal propensity to develop IgE antibodies against common environmental allergens. It is also defined as the leading clinical correlation of increased IgE levels and may typically manifest in the form of atopic eczema (atopic dermatitis), allergic rhinitis, allergic conjunctivitis, and allergic asthma. Atopy and allergy are not synonyms, in that atopic individuals may not necessarily develop one or more allergic diseases against specific allergens [3,11].

Over the past decades, numerous investigations of varying types have suggested the existence of possible links between atopy/allergic inflammation, high serum IgE, coronary artery atherosclerosis development, and acute coronary syndromes. Most of these studies were performed on live subjects, while there is a great paucity in the postmortem/forensic literature on this topic [12].

The first ever study to assess a potential relationship between total IgE and ischemic heart disease was published by Criqui et al. [13]. These authors performed a cross-sectional, population-based study including 262 men and 315 women aged between 38 and 82 years. These researchers found that men with a previous history of acute myocardial infarction had increased concentrations of IgE in serum.

Another study was then carried out by Szczeklik et al. [8] that included 116 consecutive patients with coronary artery disease. 100 cases consisted of patients with recent acute myocardial infarction, and 16 cases of individuals with acute coronary insufficiency without any evidence of recent myocardial infarction. They found that patients with acute myocardial infarction had increased levels of IgE on hospital admission and that IgE continued to rise during the first week after infarction. In addition, patients with high initial IgE levels (higher than $200 \mathrm{IU} / \mathrm{ml}$ ) prior to infarction had greater increases in lgE levels after infarction. These cases also had lower severe complication frequency than those with initial IgE levels below $200 \mathrm{IU} / \mathrm{l}$, possible suggesting that patients with high IgE levels might be protected against complications of infarction. Lastly, in individuals with acute coronary insufficiency without any evidence of recent myocardial infarction, serum IgE remained unchanged.

The findings obtained by Szczeklik et al. [8] were subsequently confirmed by Kormaz et al. [14] and Erdogan et al. [15], among others. These authors had analogous results in individuals with myocardial infarction and unstable angina pectoris.

Subsequent investigations by Szczeklik et al. [16-23] found significantly increased total serum IgE levels in patients with sudden cardiac arrest upon hospital admission (compared to patients without cardiac arrest upon admission), as well as increased total serum lgE levels after coronary artery bypass grafting. Increased serum total IgE in these patients has been suggested as the consequence of inflammatory reactions to coronary artery or myocardial damage. However, the authors explained that atopic patients prone to IgEmediated mast cell activation might appear to be protected against sudden cardiac death after myocardial infarction due to a mild haemostatic imbalance, similar to that produced by aspirin. This imbalance would be shown by moderately longer bleeding times, depressed platelet aggregability, and delayed thrombin generation in clotting blood. The late appearance of this latter would delay clot formation inside a critically obstructed coronary artery and these mechanisms, among others, might thus protect atopic patients against sudden cardiac death associated with occlusive coronary thrombosis.

It must be highlighted that data on haemostatic alterations in atopic subjects are sparse and somewhat conflicting. Bleeding time may be prolonged in patients with atopic diseases, possibly associated to complex heparin-tryptase released by activated mast cells that may reduce platelet activity and exert anticoagulant properties. On the other hand, mast cells produce and secrete mediators that might have prothrombotic properties. This might result in reduced anti-coagulant activity at the inflammation site. Nastalek et al. [6] performed a study involving 130 consecutive patients suffering from atopic dermatitis in order to investigate fibrin clot characteristics. The authors observed reduced clot permeability, prolonged fibrinolysis time, faster fibrin polymerization, higher thrombotic mass, and thicker fibers in these patients. These features are known to be linked to prothrombotic tendencies. 
Despite exhaustive studies carried out by several research teams, the relationship between atopy, allergy, total serum IgE, atherosclerosis, coronary artery disease, and acute coronary syndromes is still far from clear [24-29].

Wang et al. [9] studied 982 subjects consecutively admitted to several hospitals with suspected chronic heart disease. These included 207 individuals with acute myocardial infarction, 255 with unstable angina, 247 with stable angina, and 273 control cases with no or less than $50 \%$ luminal coronary artery narrowing. These authors observed higher total serum IgE levels in those with coronary artery disease compared to unaffected individuals. The highest levels of IgE were measured in patients with myocardial infarction, followed by those with unstable angina, and then by those with stable angina.

Sinkiewicz et al. [30] measured total serum IgE in a study population including 80 patients with acute myocardial infarction, 58 patients with troponin-negative acute coronary syndrome, 57 patients with stable angina pectoris with negative personal and family history of allergies, as well as in a control population including 39 healthy, age-matched individuals. They observed significantly increased concentrations of serum IgE in patients suffering from any form of ischemic heart disease, compared to the control group of healthy individuals. Changes in IgE serum levels on the first, seventh, fourteenth, and fortieth day post myocardial infarction did not show any significant difference. A gender difference was observed inasmuch as serum lgE levels were significantly higher in males with acute myocardial infarction than females.

Jaramillo et al. [1] carried out a retrospective study on a U.S. population and analyzed data of allergen-specific and total IgE obtained from 4002 adult participants. The aim of the study was to investigate the relationship between atopy and past myocardial infarction. These authors found that serum IgE levels were inversely related to myocardial infarction, independent of multiple coronary risk factors.

Non-significant differences in serum IgE were noticed by Koltowski et al. [31] in a study that compared 52 consecutive patients with ST segment elevation myocardial infarction (34 individuals) and unstable angina or myocardial infarction with no ST segment elevation (18 individuals).

Further evidence supporting a possible relationship between atopy, allergy, atheromatous disease, and acute cardiovascular events come from the literature on the so-called Kounis syndrome, which combines acute coronary syndromes with conditions associated with mast cell activation [12,32].

Kounis syndrome is defined as the occurrence of acute coronary syndromes (coronary spasm, acute myocardial infarction, and stent thrombosis) with conditions associated with mast cell and platelet activation, involving interrelated and interacting inflammatory cells such as macrophages and T-lymphocytes. Kounis syndrome occurs in the setting of hypersensitivity reactions and is caused by the inflammatory mediators released by the involved inflammatory cells during a reaction. Although mast cells are numerically inferior in this inflammatory cascade, it has been emphasized that they play a decisive role in the process. Indeed, mediators released by activated mast cells can induce coronary artery spasm, plaque rupture, and thrombosis, leading to a clinical picture of Kounis syndrome. On the other hand, all the cells involved in the process activate and co-activate each other, creating a sort of "vicious circle of inflammation" [12,33-42].

Three variants of Kounis syndrome have been described thus far: in the type I variant, coronary spasm in normal or nearly normal coronary arteries associated with hypersensitivity reaction can progress to myocardial infarction. In type II variant, preexisting atheromatous disease combined with hypersensitivity reactions can result in acute myocardial infarction. In type III variant, hypersensitivity reactions in individuals with any implanted coronary stent component can result in intrastent thrombosis [31].

Kounis syndrome has been postulated to represent a final mast cell-related trigger pathway, possibly shared between allergic and non-allergic coronary syndromes. This hypothesis would be supported by several lines of evidence as well as by the fact that activated mast cells have been found in the shoulder regions of atheromatous lesions and actual plaque erosion or rupture sites in patients after acute coronary syndrome or myocardial infarction $[12,43]$.

Still, it remains unclear and unexplained whether the elevated IgE levels found in individuals with acute coronary syndromes are indicative of their participation coronary 
event development or, on the contrary, they are increased following the inflammatory reaction sustained by tissues damaged during the event. In essence, the question remains as to whether increased IgE levels cause acute coronary events or are caused by them [10]. This study was carried out to examine IgE and tryptase in the deceased population. Our findings are clearly in agreement with data previously reported in both clinical and forensic literature on this topic, i.e. that a portion of acute coronary syndromes and coronary deaths characterized by coronary atherosclerotic lesions of various severities (ranging from simple lipid plaques to ruptured plaques with superimposed acute thrombi and myocardial infarction) are also characterized by increased total IgE levels (in an apparently higher proportion than those observed in individuals with other causes of death) and increased mast cell tryptase levels (higher than the clinical reference values, in the absence of any signs suggesting anaphylaxis as the cause of death).

We wish to emphasize that increased postmortem serum total IgE and mast cell tryptase (possible suggesting mast cell activation preceding acute coronary syndrome) cannot be systematically observed in coronary atherosclerosis disease, at least where the postmortem setting is concerned, as previously highlighted by other research teams [5, 43].

That notwithstanding, the findings summarized in this paper seem to provide further confirmation of the necessity of combining morphological investigations (focusing on the heart and coronary arteries) with biochemical analyses (focusing on mast cell tryptase and total IgE) in all those deaths characterized by coronary atherosclerosis disease of varying importance that forensic pathologists may routinely encounter. This combined approach could be of interest in forensic pathology and useful in considering the role allergic inflammation may play in the causal process of death in specific (cardiac) situations.

References

1) Jaramillo R, Cohn RD, Crockett PW, Gowdy KM, Zeldin DC, Fessler MB. Relation between objective measures of atopy and myocardial infarction in United States. $J$ Allergy Clin Immunol 2013;131:405-11.

2) Crans Yoon AM, Chiu V, Rana JS, Sheikh J. Association of allergic rhinitis, coronary heart disease, cerebrovascular disease, and all-cause mortality. Ann Allergy Asthma Immunol 2016;117:359-64.

3) Lippi G, Cervellin G, Sanchis-Gomar F. Immunoglobin E (IgE) and ischemic heart disease. Which came first, the chicken or the egg? Ann Med 2014;46:456-63.

4) Kovanen $P$, Kaartinen $M$, Paavonen $T$. Infiltrates of activated mast cells at the site of coronary atheromatous erosion or rupture in myocardial infarction. Circulation 1995,92:1084-8.

5) Edston E, van Hage-Hamsten M. Immunoglobin E, mast cell-specific tryptase and the complement system in sudden death from coronary artery thrombosis. Int $J$ Cardiol 1995;52:77.81.

6) Nastalek M, Wojas-Pelc A, Undas A. Plasma fibrin clot properties in atopic dermatitis : link between thrombosis and atopy. J Thromb Thrombolysis 2010;30:121-6.

7) Kovanen PT. Mast cells and degradation of pericellular and extracellular matrices: potential contributions to erosion, rupture and intraplaque haemorrhage of atherosclerotic plaques. Biochem Soc Trans 2007;35:857-61.

8) Szczeklik A, Sladek K, Szczerba A, Dropinski J. Serum immunoglobulin E response to myocardial infarction. Circulation 1988;77:1245-9. 
9) Wang J, Cheng X, Xiang MX, Alanne-Kinnunen $M$, Wang JA, Chen $H$, et al. IgE stimulates human and mouse arterial cell aptotosis nd cytokine expression and promotes atherogenesis in Apoe-/ - mice. J Clin Invest 2011;121:3564-77.

10) Kounis NG, Hahalis G. Serum IgE levels in coronary artery disease. Atherosclerosis 2016;251;498-500.

11) Skaaby $T$, Husemoen LL, Thuesen BH, Jeppesen J, Linneberg A. The association of atopy with incidence of ischemic heart disease, stroke, and diabetes. Endocrine 2015;48:541-50.

12) Potaczek DP. Links between allergy and cardiovascular or hemostatic system. Int J Cardiol 2014;70:278-85.

13) Criqui MH, Lee ER, Hamburger RN, Klauber MR, Coughlin SS. IgE and cardiovascular disease. Results from a population-based study. Am J Med 1987;82:964-8

14) Korkmaz ME, Oto A, Saraçlar Y, Oram E, Oram A, Ugurlu S, et al. Levels of IgE in the serum of patients with coronary arterial disease. Int J Cardiol 1991;31:199-204.

15) Erdogan O, Gul C, Altun A, Ozbay G. Increased immunoglobulin E response in acute coronary syndrome. Angiology 2003:54:73-9.

16) Szczeklik A, Dropinski j, P. Gora PF. Serum immunoglobin E and sudden cardiac arrest during myocardial infarction. Coron Artery Dis 1993,4:1029-2.

17) Szczeklik A, Milner PC, Birch J, Watkins J, Martin J. Prolonged bleeding time, reduced platelet aggregation, altered PAF-acether sensitivity and increased platelet mass are a trait of asthma and fever. Thromb Haemost 1986;56:283-7.

18) Szczeklik A, Jawien J. Possible role of IgE in acute phase response. Allergy 1997;52:1149-50.

19) Szczeklik A, Jawien J. Immunoglobulin $E$ in acute phase response to surgical stress. Clin Exp Allergy 1996; 26:303-7.

20) Szczeklik A, Jawien J, Stadler BM, Radwan J, Piwowarska W, Dziatkowiak A. Possible relationship between interleukin- 6 and response of immunoglobulin $E$ to surgical trauma. Ann N Y Acad Sci 1995;762;477-9.

21) Szczeklik A. Atopy and sudden cardiac death. Lancet 2000;355:2254.

22) Szczeklik A, Schmitz-Schumann M, Krzanowski M, Virchow C Sr. Delayed generation of thrombin in clotting blood of topic patients with hay fever and asthma. Clin Exp Allergy 1991;21:411-5.

23) Szczeklik A, Kranowski M, Nizankowska E, Musial J. Bleeding time and PAF-acetherinduced platelet aggregation in atopy. Agents Actions Suppl 1987;21:145-50.

24) Sinkiewicz W, Blazejewski J, Bujak R, Zekanowska E, Sobanski P, Kubica J, et al. Immunoglobulin $\mathrm{E}$ as a marker of the atherothrombotic process in patients with acute myocardial infarction. Cardiol J 2007;14:266-73.

25) Guo X, Yuan S, Liu Y, Zeng Y, Xie H, Liu Z, et al. Serum IgE levels are associated with coronary artery disease severity. Atherosclerosis 2016;251:355-60.

26) Langer RD, Criqui MH, Feigelson HS, McCann TJ, Hamburger RN. IgE predicts future myocardial infarction in men. J Clin Epidemiol 1996;49:203-9. 
27) Jong GP, Wang YF, Tsai FJ, Tsai $\mathrm{CH}$, Wu CL, Liu RH, et al. Immunoglobulin $\mathrm{E}$ and matrix metalloproteinase- 9 in patients with different stages of coronary artery disease. Chin J Psysiol 2007;50:277-82.

28) Kovanen PT, Mänttäri M, Palosuo T, Manninen V, Aho K. Prediction of myocardial infarction in dyslipidemic men by elevated levels of imunoglobulin classe A, E and G, but not M. Arch Intern Med 1998;158:1434-9.

29) Shiue I. Are higher serum IgE concentrations associated with adult cardiovascular disease? Int J Cardiol 2013;168:1580-1.

30) Sinkiewicz W, Blazejewski J, Bujak R, Kubica J, Dudziak J. Immunoglobulin E in patients with ischemic heart disease. Cardiol J 2008;15:122-8.

31) Koltowski L, Filipiak KJ, Rdzanek A, Stepien V, Tarchalska-Krynska B, Opolski G. IgM, IgG and inflammation markers serum concentrations in patients with acute coronary syndrome. Kardiol Pol 2012;70:1023-8.

32) Kounis NG, Kounis GN, Soufras GD, Lianas D, Patsouras N. Postmortem diagnosis of drug-induced anaphylactic death: Kounis syndrome and hypersensitivity myocarditis are the likely culprit in death of severe anaphylactic reactions. J Forensic Leg Med 2016;40:40-1.

33) Kounis NG. Kounis syndrome: an update on epidemiology, pathogenesis, diagnosis, and therapeutic management. Clin Chem Lab Med 2016;54:1545-59.

34) Kounis NG, Soufras GD, Kounis GN, Hahalis G. Suicidal anaphylactic death: is Kounis anaphylaxis associated syndrome the cause? Forensic Sci Int 2013;232:e42-3.

35) Kounis NG, Soufras GD, Hahalis G. Accumulation of eosinophils, mast cells, and basophils in the spleen and the coronary arteries in anaphylactic deaths: is the Kounis hypersensitivity associated syndrome present? Forensic Sci Med Pathol 2014;10:150-1.

36) Kounis NG, Soufras GD. Kounis syndrome. A primary cause for the anaphylactic shock. Cardiol J 2014;21:102-3.

37) Kounis NG, Soufras GD, Hahalis G. Anaphylactic shock: Kounis hypersensitivityassociated syndrome seems to be the primary cause. N Am J Med Sci 2013;5:631-6.

38) Kounis NG. Coronary hypersensitivity disorder: the Kounis syndrome. Clin Ther 2013;35:563-71.

39) Kounis NG, Koniari I, Roumeliotis A, Tsigas G, Soufras G, Grapsas N, et al. Thrombotic responses to coronary stents, bioresorbable scaffolds and the Kounis hypersensitivity-associated acute thrombotic syndrome. J Thor Dis 2017;9:1155-64.

40) Biteker M. Current understanding of Kounis syndrome. Expert Rev Clin Immunol 2010;6:777-88.

41) Cross B, Choudhury TR, Hindle M, Galasko G. Wasp sting induced STEMI with complete coronary artery occlusion: a case of Kounis syndrome. BMJ Case Rep 2017 pii:bcr-2017-221256.

42) Gunawardena MD, Weerasinghe A, Herath J, Amarasena N. Myocardial infarction associated with eosinophilia and plasma extravasion at multiple sites. A variant of Kounis syndrome. BMJ Case Rep 2015 pii:bcr201407987. 
43) Palmiere C, Comment L, Vilarino R, Mangin P, Reggiani Bonetti L. Measurement of Btriptase in postmortem serum in cardiac deaths. J Forensic Leg Med 2014;23:12-8. 